$\mathrm{BMB}$

\title{
Clinical significance linked to functional defects in bone morphogenetic protein type 2 receptor, BMPR2
}

\author{
Myung-Jin Kim", Seon Young Park ${ }_{*}^{\#}$ Hae Ryung Chang, Eun Young Jung, Anudari Munkhjargal, Jong-Seok Lim, \\ Myeong-Sok Lee \& Yonghwan Kim ${ }^{*}$ \\ Department of Biological Sciences, Sookmyung Women’s University, Seoul 04310, Korea
}

Bone morphogenetic protein type 2 receptor (BMPR2) is one of the transforming growth factor- $\beta$ (TGF- $\beta$ ) superfamily receptors, performing diverse roles during embryonic development, vasculogenesis, and osteogenesis. Human BMPR2 consists of 1,038 amino acids, and contains functionally conserved extracellular, transmembrane, kinase, and C-terminal cytoplasmic domains. Bone morphogenetic proteins (BMPs) engage the tetrameric complex, composed of BMPR2 and its corresponding type 1 receptors, which initiates SMAD proteins-mediated signal transduction leading to the expression of target genes implicated in the development or differentiation of the embryo, organs and bones. In particular, genetic alterations of BMPR2 gene are associated with several clinical disorders, including representative pulmonary arterial hypertension, cancers, and metabolic diseases, thus demonstrating the physiological importance of BMPR2. In this mini review, we summarize recent findings regarding the molecular basis of BMPR2 functions in BMP signaling, and the versatile roles of BMPR2. In addition, various aspects of experimentally validated pathogenic mutations of BMPR2 and the linked human diseases will also be discussed, which are important in clinical settings for diagnostics and treatment. [BMB Reports 2017; 50(6): 308-317]

\section{INTRODUCTION}

Bone morphogenetic protein type 2 receptor (BMPR2) is one of the transforming growth factor- $\beta$ (TGF- $\beta$ ) superfamily receptors, which is widely expressed in various tissues and organs, including pulmonary vascular endothelium, pulmonary vascular smooth muscle, cerebellum, hippocampus,

*Corresponding author. Tel: +82-2-710-9552; Fax: +82-2-20777322; E-mail: yhkim@sookmyung.ac.kr

${ }^{\#}$ These authors contributed equally to this work.

https://doi.org/10.5483/BMBRep.2017.50.6.059

Received 9 April 2017

Keywords: BMPR2, Embryogenesis, Mutation, Osteogenesis, Pathogenesis thyroid gland, adrenal gland, heart, liver, pancreas and kidney (1-8). Previous reports state that BMPR2 serves as a type 2 receptor for bone morphogenetic protein (BMP) ligands in mammals, and the engagement of specific BMPs to the BMPR2 and corresponding type 1 receptors plays important roles in osteogenesis, cell growth, cell differentiation, and embryonic development. Knockout of $B M P R 2$ gene is fatal for embryonic development, and conditional BMPR2 knockout mice in uterine decidua revealed that BMPR2 is essential for post-implantation and fertility (9). To date, at least 20 BMPs, seven type 1 receptors (ALK1-7), and four type 2 receptors (BMPR2, ACVR1, ACVR1b, and TGF $\beta R 1$ ) have been identified in mammals $(10,11)$. Among the 20 BMPs, BMP2, 4, 6, and 7 have been reported to engage BMPR2 and its associated type 1 receptors, and the ligand-receptor combination is likely to determine the physiological roles of BMPR2 (12). Since the extracellular signal triggered by BMPs is transmitted into the cytoplasm by formation of heteromeric receptor complex, the disruption of BMP receptors by genetic alterations results in various phenotypic abnormalities. Indeed, causative mutations in the BMPR2 gene have been reported in patients present with pulmonary arterial hypertension (PAH), chronic obstructive pulmonary disease (COPD), hereditary hemorrhagic telangiectasia (HHT), prostatic neoplasms, colorectal cancer, and obesity (12-16). In particular, BMPR2 variants in PAH patients have been extensively identified, and pathogenicity of some BMPR2 mutations has been validated by in vitro functional assays. However, growing evidences have shown that the mutations in BMPR2 gene are also implicated in other diseases, which might be due to the loss of BMPR2 functions in specific combination with the ligands, and its type 1 binding partners. In this review, we summarize functional roles of BMPR2 in diverse molecular pathways. In addition, we discuss the identified $B M P R 2$ variants and the resultant physiological disorders with experimentally validated cases.

\section{FACTORS INVOLVED IN THE BMPR2-MEDIATED SIGNALING CASCADE}

\section{BMPs}

In mammals, BMPs are part of the TGF- $\beta$ superfamily which is composed of 33 proteins, comprising of TGF- $\beta$ s, activins,

ISSN: 1976-670X (electronic edition)

Copyright (C) 2017 by the The Korean Society for Biochemistry and Molecular Biology

(c) This is an open-access article distributed under the terms of the Creative Commons Attribution Non-Commercial License (http://creativecommons.org/licenses/by-nc/4.0) which permits unrestricted non-commercial use, distribution, and reproduction in any medium, provided the original work is properly cited. 
inhibins, nodal, lefty, Growth and differentiation factors (GDFs), anti-müllerian hormone (AMH), and BMPs (17). BMPs were initially discovered as factors that induce the ectopic formation of cartilage and bone in rats (18). It was later determined that BMPs in mammals have multiple roles in skeletal development, bone homeostasis, and tissue regeneration by triggering signal transduction via a complex composed of distinct transmembrane serine/threonine kinase receptors, BMPR1 and BMPR2. In addition, BMPs possess potent osteogenic activities, enabling the in vivo generation of ectopic bone formation (19). To date, at least 20 BMPs have been identified in mammals. Among them, BMP2, 4, 6, 7, and 9 are associated with high osteogenic activity (20). BMP2 is especially an indispensable factor for osteogenesis, and is being studied actively in human clinical uses for bone regeneration, and therapeutic trials of pathogenesis related to bone $(21,22)$. BMP2 also acts as a major factor in endochondral bone development, and induces expression of osteoblastic differentiation markers including alkaline phosphatase (ALP), osteocalcin, and RUNX2 (23). Similarly, BMP4 and BMP7 are responsible for the formation and repair of endochondral bone (24). BMP5 is required for initiation of normal skeletal development, and BMP9, together with vascular endothelial growth factor A (VEGFA), effectively stimulates ectopic bone formation $(25,26)$. In contrast, BMP3 knockout mice studies showed that BMP3 plays a role as a negative controller of bone development (27).

BMPs also play crucial roles in the establishment of basic embryonic body plans, which includes the initial vertebrate gastrulation, mesoderm, somite, neural patterning, development of limb and skeleton, and organogenesis $(28,29)$. It was reported that BMP2-deficient embryos exhibit defects in the development of heart, the first organ to be formed during embryogenesis. Further studies showed that the heart defect is due to the lack of interaction between ectodermal and mesodermal cells during development, demonstrating essential roles of BMP2 in organ development (30). Similar studies found that BMP4 exerts the formation of mesoderm during early gastrulation, and BMP7 functions in the organogenesis of heart, kidney, and eye (31-33).

The significant roles of BMPs in vascular development have been emphasized over the years, which have been verified in vascular disorders including $\mathrm{HHT}$ and $\mathrm{PAH}$. For example, BMP9 and BMP10, as core cardiac-derived factors, are highly expressed in the heart. Experiments have shown that BMP9 and BMP10 modulate the pulmonary vascular function by engaging BMP receptor complex composed of ALK1 (ACVRL1), BMPR2, and Endoglin (ENG) to activate SMAD1/5/8-mediated signal transduction. Genetic alterations of genes encoding the complex, leading to attenuation of BMP9/10 signaling and thus inducing unbalanced angiogenetic responses, have been identified in patients present with HHT or PAH (34). In particular, BMPR2 variants in $\mathrm{PAH}$ patients have been extensively identified in the past decade, which will be discussed below.

\section{BMP receptors}

BMPs initiate SMAD protein-mediated signaling by binding to a hetero-dimeric receptor complex. There are three BMP type 1 receptors, ACVR1 (also known as activin receptor-like kinase 2, ALK2), BMPR1a (ALK3), and BMPR1b (ALK6), and three BMP type 2 receptors, BMPR2, ACVR2a (ActR2a), and ACVR2b (ActR2b). Both type 1 and type 2 BMP receptors share common membrane receptor structures, such as a short extracellular ligand binding domain, a single membranespanning domain, and an intracellular serine/threonine kinase domain. The type 1 receptors carry two additional motifs, a glycine/serine-rich region preceding the kinase domain (GSbox) and a short region of eight amino acids (denoted as L45 loop) within its kinase domain $(35,36)$. An unusual property of type 1 receptors comes from a highly conserved GS motif which regulates the kinase activity of the receptor (37). The three type 1 receptors have redundant roles in skeletal development. BMPR1a and BMPR1b are structurally similar to each other, and have functionally akin features in chondrocyte condensations and developing skeletons. However, ACVR1 possesses a unique function to induce ectopic osteoblastogenesis through the activation of SMAD1/5 signaling $(38,39)$.

Type 2 BMP receptors are expressed in diverse tissues. Although all type 2 receptors have similar structures, BMPR2 has a unique 508-amino acid long C-terminal tail following the kinase domain. Long and short forms of BMPR2 have been isolated, where the short isoform is the splice variant lacking exon 12, coding most of the C-terminal tail. Similar to the long BMPR2 variant, the short form of BMPR2 is broadly expressed (12). Recently, it was reported that a single di-leucine motif located in the long C-terminal BMPR2 facilitates faster clathrin-mediated endocytosis than the short form. Further studies showed that enhanced expression of the short BMPR2 at plasma membrane led to increased activation of SMAD protein-mediated signaling, suggesting that the C-terminal region may be modulating the activity of BMPR2 (40). The physiological importance of the C-terminal region of BMPR2 has been emphasized by the identification of C-terminal truncation mutations of BMPR2 gene in familial primary pulmonary hypertension $(\mathrm{PPH})$ patients, although the exact molecular function of the C-terminal region of BMPR2 remains elusive (41-43). Different BMPs engage different combinations of BMPR2 and its type 1 receptors, which is summarized in Fig. 1. To understand the pathophysiology of the disease due to BMPR2 mutations and eventually develop novel therapeutics, it would be important to understand the different biological functions of BMPR2 in response to different BMP ligands.

\section{Ligand-receptor oligomerization and signaling}

The BMPs, initially expressed as large inactive precursors, are commonly dimerized either with itself or with a different 


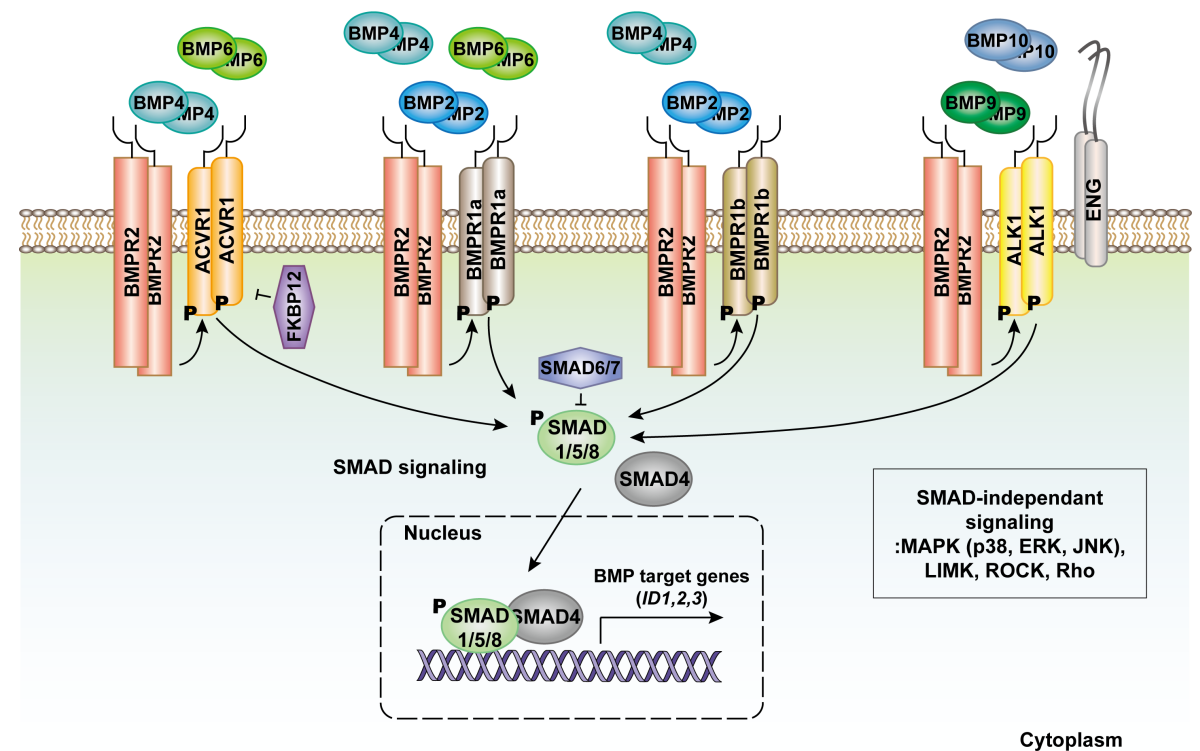

Fig. 1. Schematic summary of BMPR2 and its associated proteins in the BMP-mediated signaling cascade. Different BMP ligands engage BMPR2 and different corresponding type 1 receptors, such as ACVR1, BMPR1a, BMPR1b and ALK1. BMP4 and BMP6 ligands bind to a receptor complex consisting of BMPR2 and ACVR1; BMP2, BMP4, and BMP6 bind to BMPR2 and BMPR1a; BMP2 and BMP4 bind to BMPR2 and BMPR1b; and BMP9 and BMP10 bind to BMPR2 and ALK1. The activity of ACVR1 is negatively regulated by interaction with FKBP12. Endoglin, as a co-receptor for ALK1, promotes the receptor complex formation. Engagement of BMP ligands triggers SMAD1/5/8 phosphorylation, which then binds to SMAD4. The SMAD complex translocates into the nucleus to induce target gene expression. SMAD6 and SMAD7, which are induced by BMP signaling bind to type 1 receptors and thus, negatively regulate BMP signaling by negative feedback loop. Some of the BMP target genes, including ID proteins, are indicated. MAPK, LIMK, ROCK, and Rho are activated by SMAD protein-independent manner.

member of BMPs. The inactive dimeric BMPs are cleaved by proteolysis to produce the small mature BMPs, which are subsequently secreted from cells to conduct biological functions (44). It was reported that BMP heterodimers, such as $\mathrm{BMP} 2 / 5, \mathrm{BMP} 2 / 6, \mathrm{BMP} 2 / 7, \mathrm{BMP} 2 \mathrm{~b} / 7$, and BMP4/7, are more potent activators of BMP signaling than the homodimers (45). For example, BMP4/7 heterodimer induces stronger activity of mesoderm than BMP4 or BMP7 homodimers in Xenopus. Similarly, BMP2/7 heterodimer has higher activity in bone regeneration than BMP2 or BMP7 homodimers in mammals (46). In addition, BMPs bind to various BMP receptors with different affinities. For instance, BMP2 and BMP4 have a higher affinity to BMPR1a and BMPR1b, but relatively low affinity to BMPR2 (47). In contrast, BMP7 preferentially binds to ACVR2a and ACVR2b, whereas it barely interacts with type 1 receptors in general (48). The different binding affinities allow to engage the ligand and receptor complex, triggering the activation of distinctive signaling pathway $(49,50)$.

Previous reports indicate that both type 1 and type 2 receptors individually form a dimer when they are associated with the BMP ligands. Therefore, active BMPs engage a tetrameric receptor complex (17). In general, while type 2 receptors are capable of binding BMP ligands on their own, the type 1 receptors are unable to interact with BMPs unless they are associated with corresponding type 2 receptors. BMPR2 weakly binds to BMPs alone, but the interaction is augmented in the presence of type 1 receptors (43). The serine/threonine kinase domain of type 2 receptors is constitutively active, but the activation of type 1 receptor kinase requires physical interaction with type 2 receptors through ligand engagement (51-54). Subsequently, the activated type 1 receptor phosphorylates R-SMAD proteins including SMAD1, SMAD5, and SMAD8, and the phosphorylated SMAD1/5/8 is associated with the common-mediator SMAD (Co-SMAD), SMAD4. The resultant SMAD complex, which functions as a transcription activator, translocalizes to the nucleus and activates the corresponding target gene expression. In contrast, the inhibitory-SMADs (I-SMADs), SMAD6 and SMAD7, are responsible for negative feedback of the signaling pathway (55). SMAD6 efficiently inhibits BMP signaling, and SMAD7 attenuates TGF- $\beta$, activin, and BMP signaling. I-SMADs disrupt the association of R-SMAD and Co-SMAD, resulting in inhibition of the expression of target genes. SMAD ubiquitin regulatory factors (Smurfs), an E3 ubiquitin ligase, leads to the ubiquitination and degradation of SMAD proteins, thereby modulating the BMP signals. Smurfs enhance the ubiquitination and degradation of type 1 receptors by interacting with SMAD7, forming a complex with type 1 receptors (56). 
Additionally, Smurf1 has shown to control BMP signaling by targeting SMAD1/5 for ubiquitination and proteasomal degradation, which leads to enhanced sensitivity to the TGF- $\beta$ signaling (57).

\section{Target gene regulation}

Phosphorylated R-SMAD and SMAD4 complex bind to the promoters of target genes, leading to gene expression. Of the various BMP target genes, ID proteins (inhibitory of differentiation) have been extensively studied. IDs are expressed ubiquitously, although the expression level is down-regulated in differentiating cells. In other studies, Ogata et al. showed that the ID genes are highly expressed in osteoblast lineages, suggesting that IDs play important roles in osteogenesis (58, 59). Activated IDs inhibit the transcription activity of the myogenesis factor, MyoD, which in turn suppresses myogenesis (53). Consistently, BMP2 inhibits myogenic differentiation in C2C12 myoblasts by inducing transcription of ID1, resulting in stimulation of osteoblast differentiation (60).

Another well characterized BMP target gene is the runtrelated transcription factor (RUNX), which promotes bone formation and hematopoiesis (61). Three RUNX isotypes (RUNX1, RUNX2, and RUNX3) have been identified. RUNX expression is induced by either SMAD-dependent or SMADindependent manner. As a major transcription factor, RUNX2 is often associated with SMAD1 or SMAD5, and both factors synergistically regulate the transcription of genes required for differentiation of mesenchymal progenitor cells into osteoblasts $(61,62)$. RUNX2 up-regulates several osteogenic markers including ALP, at the early osteogenic stage, and osteocalcin and osteopontin, at the late stage (63). Osterix, another transcription factor induced by BMP signaling, mediates the differentiation of mesenchymal stem cells (MSCs) into bone cells (64). Other transcription factors associated with SMAD signaling contain TBX20 and VEGF. TBX20 has an important role in cardiac development, and VEGF is the main factor involved in angiogenesis.

\section{ROLES OF BMPR2 DURING THE EMBRYOGENIC DEVELOPMENT AND BONE DIFFERENTIATION}

\section{BMPR2 in embryogenesis}

Although initially identified as a factor for regulating the chondrogenic and osteogenic differentiation, accumulated studies demonstrate that BMPR2 plays important roles in early embryonic development (65). The expression levels of BMPR2 fluctuate during embryonic stages, although it is reported that basal level of BMPR2 expression is sustained in most tissues throughout development. BMPR2 expression is relatively low during early stages of heart development, but the expression level continuously increases with gradual embryonic development, especially in the anteriormost telencephalon, branchial arches, limb bud, and tail tip mesoderm. In the later stages, BMPR2 expression is specifically high in neuroectoderm of the mouth anlagen (66). In this regard, BMPR2 acts as an essential regulator in the developing organs and tissues. BMPR2 deficient mice consistently exhibit severe embryonic lethality prior to gastrulation. More specifically, BMPR2 null murine embryos induce collapse of the gastrulation and mesoderm formation, which are similarly observed in BMP4, ALK3, and SMAD4 null embryos. In addition, overexpression of dominant negative BMPR2 brings about abnormal mesoderm formation and patterning. Epiblast differentiation in the embryo and the anterior-posterior (A-P) axis were shown to be abnormal in $B M P R 2$ knockout mice, although the differentiation of visceral endoderm was relatively normal (65). Taken together, these findings suggest that BMPs-BMPR2-mediated signal transduction is critical for diverse tissue and organ development.

\section{BMPR2 in vascular development}

BMP signaling enhances the endothelial specification, subsequent venous differentiation and angiogenesis during embryonic development, thereby maintaining the vascular homeostasis $(30,67)$. Notably, BMPR2, as a component of the BMP signaling transduction, is prominently expressed in the vascular endothelium and smooth muscle layer of the pulmonary vasculature in normal lung, but is poorly expressed in the airway and arterial smooth muscle (68). BMPR2 is expressed in human microvascular endothelial cells (HMVECs), human umbilical vein endothelial cells (HUVECs), and aortic endothelial cells, highlighting that BMPR2-mediated signaling cascade plays important roles in vascular development (69).

It was reported that BMP2 is expressed in a variety of cancers. To understand the functional relevance of the BMP2 in cancer cells, BMP2-overexpressed A549 cells were injected in nude mice, and it was observed that BMP2-mediated signaling was involved in tumor angiogenesis (70). In addition, Wiley et al. showed that BMP2-BMPR2-mediated signaling regulates sprouting angiogenesis from the axial vein in zebrafish development, demonstrating that BMPR2-dependent signaling promotes endothelial cell proliferation and angiogenesis (71). To promote human pulmonary arterial endothelial cell (HPAEC) survival and proliferation through ERK1/2 activation, BMPR2 is associated with the canonical WNT signaling pathway, which also stimulates non-canonical RhoA-Rac1 pathway to induce endothelial cell migration (72). Defects in BMPR2 cause abnormal vascular remodeling. Pulmonary endothelium in BMPR2 knockout mice has shown to the inclination of $\mathrm{PAH}$, which is characterized by reduced lumen diameter and decreased vasodilation ability resulting from increased proliferation of vascular smooth muscle cells (VSMCs) and excess deposition of extracellular matrix (ECM) in the vessel walls (73). Similarly, BMPR2 mutant mice lacking C-terminal tail in the pulmonary artery smooth muscle cells (PASMCs) have a PAH-like predisposition. Restoration of $B M P R 2$ in mice exposed to chronic hypoxia induces activity of BMP-SMAD1/5 signaling, followed by decline of vascular remodeling, implying a functional imbalance of BMP signaling 
$(74,75)$. Therefore, BMPR2 expression in ECs and SMCs has important roles in the maintenance of vascular integrity of the pulmonary arteries (76).

\section{BMPR2 in Osteogenesis}

Although biological functions of BMPR2 have been observed in bone formation, the regulatory role of BMPR2 in chondrogenesis and osteogenesis is not yet clearly demonstrated. BMPR2 is involved in osteoblast differentiation, and is also an important mediator in bone formation and skeletal development during fracture healing, otospongiosis, and osteosclerosis (77-79). During bone development, BMPR2 and its ligands induce the differentiation of mesenchymal stem cells toward osteoblastic lineage, which promotes the maturation of osteoblasts (80). To elucidate the underlying mechanism of osteogenesis, fibroblast, MSC, and myoblast have been utilized to induce osteogenic differentiation, as naïve osteoblasts are difficult to isolate, manipulate, and expand in vitro. Briefly, the differentiation process of osteoblasts is classified into two steps: first, is the differentiation of MSCs into osteoblast progenitors, and second, is the maturation of osteoblast progenitors into osteoblasts, presenting the various phenotypes of cells organizing bone (81). For the process of differentiation, useful alternative cells are the dermal fibroblasts, which are easily available and expandable (82). In the late 1980s, C3H10T1/2, a pluripotent fibroblastic and MSC, was cloned, which can differentiate into a myogenic lineage by introducing genes of the muscle-specific regulatory factors, including MyoD, myogenin, and Myf-5 (83-86). Since then, Katagiri et al. reported that the $\mathrm{C} 3 \mathrm{H} 10 \mathrm{~T} 1 / 2$ cells can also be differentiated into the osteoblast-like cells by stimulation of recombinant human BMP2 protein. They also verified the osteogenic function of BMP2 using myoblastic $\mathrm{C} 2 \mathrm{C} 12$ cells, in which treatment of BMP2 inhibits myotube formation from $\mathrm{C} 2 \mathrm{C} 12$. Instead, the cells start to induce ID1 expression so that the myoblasts can undergo differentiation toward osteoblast lineage by BMP2 $(81,87)$. The osteogenic activity of BMPs is dependent on type 2 receptors. For instance, Wu et al. showed that both BMPR2 and ACVR2 are responsible for osteogenic differentiation of $\mathrm{C} 3 \mathrm{H} 10 \mathrm{~T} 1 / 2$ cells with BMP9 treatment (88). Taken together, these findings demonstrate that BMPR2mediated signal transduction plays a critical role in skeletal development.

\section{PATHOGENIC MUTATION OF BMPR2}

The importance of the diverse BMPR2 functions has been highlighted by the identification of potential causative BMPR2 gene variants in patients present with diseases, including $\mathrm{PAH}$, cancers and obesity. BMPR2 gene consists of 13 exons, which code for the typical 4 domains described above. Identified genetic alterations of $B M P R 2$ gene lead to missense, nonsense, frameshift, truncation, and splice site mutations, which supposedly results in the loss of BMPR2-mediated signaling with some exceptions. BMPR2 mutations have been extensively determined in patients present with PAH characterized by elevated pulmonary arterial pressure (89). It was reported that PAH patients with BMPR2 mutations usually have a worse prognosis than patients with wildtype BMPR2. Not every $B M P R 2$ mutations are validated functionally, either with the patient-driven cells or with other in vitro assays. In this section, we summarize the most up-to-date BMPR2 mutations in diverse diseases, with emphasis on experimentally validated BMPR2 mutations.

\section{BMPR2 mutations in PAH}

Accumulating body of evidence demonstrates that BMPR2 mutations are strongly associated with hereditary PAH (HPAH). BMPR2 mutations have been determined in $75 \%$ of HPAH patients and also in $15 \%$ of idiopathic PAH (IPAH) patients. HPAH has been defined as an autosomal-dominant disorder, and thus pathophysiology of the BMPR2 mutation in the PAH can be explained by haploinsufficiency. It is worth noting that only $20 \%$ of people who have the BMPR2 mutation develop $\mathrm{HPAH}$, suggesting that there are additional factors, including genetic alterations or environmental agents, required for the development of the disease. Interestingly, it was reported that the wildtype BMPR2 transcripts and protein expression levels are impaired in PAH cells with BMPR2 mutation in the other allele. This observation might explain the reduction of BMPR2 expression in PAH patient-derived cells, although the underlying molecular basis remains elusive. It is still not clear why the BMPR2 mutation carriers eventually develop PAH. One hypothesis is that decreased BMP signal transduction might lead to hyperactivation of the TGF- $\beta$ signaling, resulting in hyperproliferation of the SMCs in pulmonary arterioles. Consistent with this idea, it was found that activation of BMP signaling indeed inhibited the smooth muscle cell proliferation. However, the underlying molecular mechanism of enhanced TGF- $\beta$ signaling cascade in response to reduced BMP activity is largely unknown. Potential causative BMPR2 mutations in PAH are basically distributed throughout the BMPR2 region, although more frequent $B M P R 2$ mutations have been identified in key functional domains, such as the ligand binding domain and kinase domain. To date, over 400 different BMPR2 mutations in PAH patients have been reported, and functional defects of some of the BMPR2 variants have been validated with patient-driven cells or in vitro functional assays. Most of the experimentally validated BMPR2 mutations show an impaired SMAD proteinmediated signaling cascade (Fig. 2). The BMPR2 mutations in PAH have been regularly updated, and the most recent update is by Machado et al. However, not all mutations are functionally validated using various techniques available in life science. As the functional validation of the BMPR2 mutations is important and informative to understand the pathophysiology of PAH, we will summarize most of the validated BMPR2 mutations in this review. 


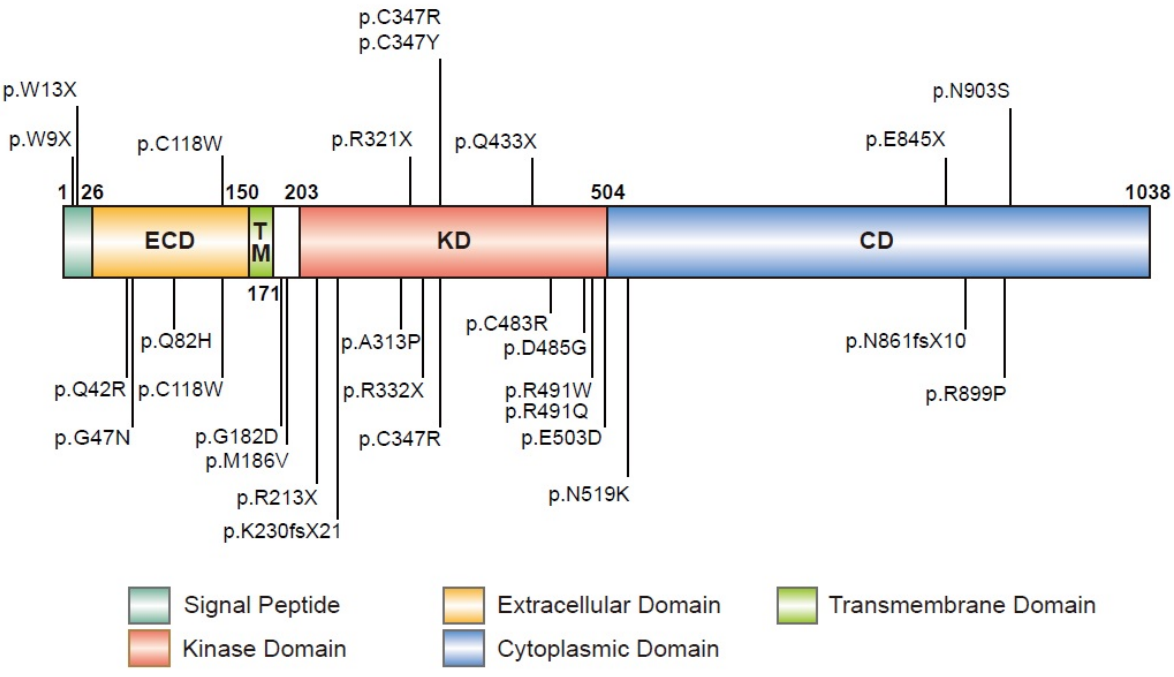

Fig. 2. Experimentally verified BMPR2 mutations are indicated on the BMPR2 gene. BMPR2 domains are indicated. Pathogenic BMPR2 mutations functionally validated in patient-derived cells are indicated above. Pathogenic BMPR2 mutations validated by in vitro functional assays are indicated below.
Yang et al. obtained PASMCs from $\mathrm{PAH}$ patients with individual BMPR2 mutation leading to p.W9X, p.C347R, p.C347Y, and p.N903S. Compared to normal PASMCs, they noticed impaired SMAD protein phosphorylation and reduced ID1 and ID2 protein expression, which are downstream targets of SMAD proteins, in BMPR2 mutant cells treated with BMPs (90). Hedges et al. employed patient-derived cultured lymphocytes to validate the function of BMPR2 p.W13X mutant. Initially, the authors presumed that the p.W13X mutant will not be expressed due to the nonsense-mediated RNA decay (NMD). However, they surprisingly found that the truncated version of BMPR2, which lost the first 151 amino acids, is expressed together with the wildtype BMPR2 from normal allele, due to the existence of a downstream Kozak sequence enabling translation re-initiation (91). The truncated version of BMPR2 is an inactive form, as the ligand binding domain is lost. Likewise, the functional assays of other BMPR2 variants listed in Fig. 2 (upper) have been performed with patientderived PASMCs and PAECs $(92,93)$. Another way to determine functionality of $B M P R 2$ variants can be accomplished by ectopic expression of BMPR2 mutants in established cell lines to measure the BMPR2-mediated signals using various experimental tools, including immunoblotting, quantitative polymerase chain reaction, reporter assay and immunostaining. For example, to determine NMD of BMPR2 variants, c.2292insA, c.2386delG, c.2620G $>$ T and c.2695C $>$ T, Nasim et al. developed a novel dual fluorescence based assay system; the successful expression of the variant will give rise to red and green fluorescence simultaneously in HEK293T cells. Indeed, the BMPR2 mutations failed to express green fluorescence, thereby indicating that the mRNA is degraded by NMD (94). In the same studies, various missense BMPR2 mutants were expressed in HEK293T cells harboring the luciferase reporter, where the luciferase expression is under the BMP responsive promoter. Using the reporter system, they were able to validate various loss of function missense mutations of $B M P R 2$ (94). Taken together, the in vitro functional assay proved that most of the causative BMPR2 mutations in PAH lead to impairment of the SMAD protein-mediated signal transduction.

\section{BMPR2 mutations in cancer}

Similar to tumor suppressor roles of TGF- $\beta$, BMPR1a and SMAD4 genes are frequently mutated in colon cancers, suggesting that BMPs-mediated signaling also has tumor suppressive functions (95). BMPR2 gene is found to be mutated or down-regulated in cancers. It was reported that BMPR2 expression level is significantly down-regulated in prostate cancer tissues. In addition, expression level of BMPR2 in the prostate cancer cells is negatively correlated with cancer grade (14). Indeed, the reduced expression level of BMPR2 showed statistically significant poor prognosis, such as cancer recurrence and worse 5 year survival (96). BMPR2 expression level is significantly abrogated in large portions of microsatellite instable (MSI) colorectal cancers. Kodach et al. initially found impaired expression of BMPR2 in MSI colon cancer cell lines including HCT116, DLD1, SW48, and LOVO, while its expression was normal in microsatellite stable (MSS) colon cancer cell lines. Reduced BMPR2 expression level is also confirmed in all the MSI positive colon cancer patient tissues tested in the study. Mutation analysis revealed that HCT116 and LOVO cell lines have BMPR2 mutation in the coding region at 7 adenine tract in exon 12 (c.1742delA), resulting in frameshift and early termination of translation. However, such a mutation has not been found in the cancer patient tissues. Instead, 11 adenine tract in $3^{\prime}$ UTR of BMPR2 gene is mutated in all the colon cancer patient tissues, which results in down-regulation of BMPR2 expression (97). Later, by analyzing public datasets, Park et al. found the 
7 adenine tract mutations in BMPR2 gene in MSI positive colorectal cancer patients (15). Taken together, these findings suggest that genetic alterations of BMPR2 gene and the resultant reduced expression of BMPR2 might be responsible for cancer development, although the underlying mechanism remains largely elusive.

\section{BMPR2 mutation in obesity}

BMP-mediated signaling has been implicated in controlling adipocyte differentiation, and it was therefore proposed that BMPR2 expression might be positively correlated to obesity (98). Indeed, Schleinitz et al. found that BMPR2 mRNA level is significantly high in both visceral and subcutaneous adipose tissue of the overweight or obese population, compared to the lean population. In an attempt to define genetic alterations in BMPR2 of overweight population, two intronic single nucleotide polymorphisms (SNPs) were identified: rs6717924 and rs13426118. Gene expression analysis showed that allele carrying rs6717924 expresses higher BMPR2 mRNA compared to the other wildtype allele. The authors proposed that transcription factor binding sites surrounding the SNPs might be responsible for the enhanced gene expression, although the molecular basis of the higher BMPR2 expression in allele carrying rs6717924 remains elusive (16).

\section{CONCLUSION AND PERSPECTIVES}

BMPR2, a receptor for the TGF- $\beta$ superfamily, was identified in the 1990s. Since then, understanding the functional roles of BMPR2 has significantly expanded our knowledge in the fields of embryonic development, vasculogenesis and osteogenesis. Identification of causative BMPR2 mutations in HPAH, IPAH and other diseases emphasizes the important physiological functions of BMPR2. In particular, it is widely accepted that functional defects of BMPR2 are implicated in the development of PAH. Indeed, most of the BMPR2 mutations identified in $\mathrm{PAH}$ are nonsense mutations, the mRNA of which get degraded by NMD. In addition, in vitro functional assays proved that most of the BMPR2 missense mutations lead to defects in SMAD protein-mediated signal transduction. Although haploinsufficiency is the common disease mechanism in PAH, significantly reduced BMPR2 transcripts have been found in patient-derived pulmonary vascular cells, suggesting that not only the BMPR2 mutated allele, but wildtype BMPR2 allele is also not expressed due to the unknown additional defects in the wildtype allele. Considering the low penetrance of HPAH, transcript level of wildtype BMPR2 allele would be the reasonable diagnostic marker for PAH. Questions to be addressed here pertain to understanding the molecular basis of the development of PAH. Advanced sequencing technology allows us to find additional causative mutations of BMPR2 in patients present with $\mathrm{PAH}$. However, only limited experimental results are currently available to understand the pathophysiology of PAH, due to the impairment of BMPR2 functions.
Understanding the disease at the molecular level is the perquisite to overcoming the disease. Other than $\mathrm{PAH}$, potential causative mutations of BMPR2 gene have been determined in cancers and obesity. Since BMPR2 plays important roles in diverse biological pathways, it would possible to identify causative BMPR2 mutations in other diseases also. Analyzing genetic alterations in human diseases is one of the best ways to understand the molecular functions of the genes. Further efforts to characterize the gene at the bench will be critical for introducing new ways to diagnosis and cure the disease in return.

\section{ACKNOWLEDGEMENTS}

We thank the members of laboratory of genome maintenance for their comments. This work was supported by the National Research Foundation of Korea (NRF) grants by the Korea government (MSIP) (NRF-2016R1A5A1011974), Genome Technology to Business Translation Program (2014M3C9A2064688), and by the Ministry of Education (2016R1A6A3A11931568). This work was also partially supported by a grant from the National R\&D program for Cancer Control, Ministry of Health \& Welfare, Republic of Korea (No. 1420090) and by the Sookmyung Women's University BK21 Plus Scholarship.

\section{CONFLICTS OF INTEREST}

The authors have no conflicting financial interests.

\section{REFERENCES}

1. Harper RL, Reynolds AM, Bonder CS and Reynolds PN (2016) BMPR2 gene therapy for PAH acts via Smad and non-Smad signalling. Respirology 21, 727-733

2. Zhang D, Mehler MF, Song Q and Kessler JA (1998) Development of bone morphogenetic protein receptors in the nervous system and possible roles in regulating trkC expression. J Neurosci 18, 3314-3326

3. Franzen A, Piek E, Westermark B, ten Dijke P and Heldin NE (1999) Expression of transforming growth factor-beta1, activin A, and their receptors in thyroid follicle cells: negative regulation of thyrocyte growth and function. Endocrinology 140, 4300-4310

4. Rege J, Nishimoto HK, Nishimoto K, Rodgers RJ, Auchus RJ and Rainey WE (2015) Bone Morphogenetic Protein-4 (BMP4): A Paracrine Regulator of Human Adrenal C19 Steroid Synthesis. Endocrinology 156, 2530-2540

5. Wu X, Sagave J, Rutkovskiy A et al (2014) Expression of bone morphogenetic protein 4 and its receptors in the remodeling heart. Life Sci 97, 145-154

6. Mayeur C, Leyton PA, Kolodziej SA, Yu B and Bloch KD (2014) BMP type II receptors have redundant roles in the regulation of hepatic hepcidin gene expression and iron metabolism. Blood 124, 2116-2123

7. Dichmann DS, Miller CP, Jensen J, Scott Heller R and Serup P (2003) Expression and misexpression of members 
of the FGF and TGFbeta families of growth factors in the developing mouse pancreas. Dev Dyn 226, 663-674

8. Bush KT, Sakurai H, Steer DL et al (2004) TGF-beta superfamily members modulate growth, branching, shaping, and patterning of the ureteric bud. Dev Biol 266, 285-298

9. Nagashima T, Li Q, Clementi C, Lydon JP, DeMayo FJ and Matzuk MM (2013) BMPR2 is required for postimplantation uterine function and pregnancy maintenance. J Clin Invest 123, 2539-2550

10. Wiley DM and Jin SW (2011) Bone Morphogenetic Protein functions as a context-dependent angiogenic cue in vertebrates. Semin Cell Dev Biol 22, 1012-1018

11. Heinecke K, Seher A, Schmitz W, Mueller TD, Sebald W and Nickel J (2009) Receptor oligomerization and beyond: a case study in bone morphogenetic proteins. BMC Biol 7, 59

12. Morrell NW (2006) Pulmonary hypertension due to BMPR2 mutation: a new paradigm for tissue remodeling? Proc Am Thorac Soc 3, 680-686

13. Rigelsky $C M$, Jennings $C$, Lehtonen $R$, Minai OA, Eng $C$ and Aldred MA (2008) BMPR2 mutation in a patient with pulmonary arterial hypertension and suspected hereditary hemorrhagic telangiectasia. Am J Med Genet A 146A, 2551-2556

14. Kim IY, Lee DH, Ahn HJ et al (2000) Expression of bone morphogenetic protein receptors type-IA, -IB and -II correlates with tumor grade in human prostate cancer tissues. Cancer Res 60, 2840-2844

15. Park SW, Hur SY, Yoo NJ and Lee SH (2010) Somatic frameshift mutations of bone morphogenic protein receptor 2 gene in gastric and colorectal cancers with microsatellite instability. APMIS 118, 824-829

16. Schleinitz D, Kloting N, Bottcher Y et al (2011) Genetic and evolutionary analyses of the human bone morphogenetic protein receptor 2 (BMPR2) in the pathophysiology of obesity. PLoS One 6, e16155

17. Wu M, Chen G and Li YP (2016) TGF-beta and BMP signaling in osteoblast, skeletal development, and bone formation, homeostasis and disease. Bone Res 4, 16009

18. Miyazono K, Kamiya Y and Morikawa M (2010) Bone morphogenetic protein receptors and signal transduction. J Biochem 147, 35-51

19. Kang Q, Sun $\mathrm{MH}$, Cheng $\mathrm{H}$ et al (2004) Characterization of the distinct orthotopic bone-forming activity of 14 BMPs using recombinant adenovirus-mediated gene delivery. Gene Ther 11, 1312-1320

20. Luu HH, Song WX, Luo X et al (2007) Distinct roles of bone morphogenetic proteins in osteogenic differentiation of mesenchymal stem cells. J Orthop Res 25, 665-677

21. Razzouk S and Sarkis R (2012) BMP-2: biological challenges to its clinical use. N Y State Dent J 78, 37-39

22. Kanakaris NK and Giannoudis PV (2008) Clinical applications of bone morphogenetic proteins: current evidence. J Surg Orthop Adv 17, 133-146

23. Sun J, Li J, Li C and Yu Y (2015) Role of bone morphogenetic protein-2 in osteogenic differentiation of mesenchymal stem cells. Mol Med Rep 12, 4230-4237

24. Hyun SJ, Han DK, Choi SH et al (2005) Effect of recombinant human bone morphogenetic protein-2, -4 , and -7 on bone formation in rat calvarial defects. J
Periodontol 76, 1667-1674

25. King JA, Marker PC, Seung KJ and Kingsley DM (1994) BMP5 and the molecular, skeletal, and soft-tissue alterations in short ear mice. Dev Biol 166, 112-122

26. Pi CJ, Liang KL, Ke ZY et al (2016) Adenovirus-mediated expression of vascular endothelial growth factor-a potentiates bone morphogenetic protein9-induced osteogenic differentiation and bone formation. Biol Chem 397, 765-775

27. Daluiski A, Engstrand T, Bahamonde ME et al (2001) Bone morphogenetic protein-3 is a negative regulator of bone density. Nat Genet 27, 84-88

28. Hogan BL (1996) Bone morphogenetic proteins in development. Curr Opin Genet Dev 6, 432-438

29. Zhao GQ (2003) Consequences of knocking out BMP signaling in the mouse. Genesis 35, 43-56

30. Zhang $\mathrm{H}$ and Bradley A (1996) Mice deficient for BMP2 are nonviable and have defects in amnion/chorion and cardiac development. Development 122, 2977-2986

31. Winnier G, Blessing M, Labosky PA and Hogan BL (1995) Bone morphogenetic protein- 4 is required for mesoderm formation and patterning in the mouse. Genes Dev 9, 2105-2116

32. Jena N, Martin-Seisdedos C, McCue P and Croce CM (1997) BMP7 null mutation in mice: developmental defects in skeleton, kidney, and eye. Exp Cell Res 230, 28-37

33. Kim RY, Robertson EJ and Solloway MJ (2001) Bmp6 and Bmp7 are required for cushion formation and septation in the developing mouse heart. Dev Biol 235, 449-466

34. Tillet E and Bailly S (2014) Emerging roles of BMP9 and BMP10 in hereditary hemorrhagic telangiectasia. Front Genet 5, 456

35. Rao SM, Ugale GM and Warad SB (2013) Bone morphogenetic proteins: periodontal regeneration. $\mathrm{N}$ Am J Med Sci 5, 161-168

36. Sieber C, Kopf J, Hiepen C and Knaus P (2009) Recent advances in BMP receptor signaling. Cytokine Growth Factor Rev 20, 343-355

37. Lin SJ, Lerch TF, Cook RW, Jardetzky TS and Woodruff TK (2006) The structural basis of TGF-beta, bone morphogenetic protein, and activin ligand binding. Reproduction $132,179-190$

38. van Dinther M, Visser N, de Gorter DJ et al (2010) ALK2 $\mathrm{R} 206 \mathrm{H}$ mutation linked to fibrodysplasia ossificans progressiva confers constitutive activity to the BMP type I receptor and sensitizes mesenchymal cells to BMPinduced osteoblast differentiation and bone formation. J Bone Miner Res 25, 1208-1215

39. Yoon BS, Ovchinnikov DA, Yoshii I, Mishina Y, Behringer RR and Lyons KM (2005) Bmpr1a and Bmpr1b have overlapping functions and are essential for chondrogenesis in vivo. Proc Natl Acad Sci U S A 102, 5062-5067

40. Amsalem AR, Marom B, Shapira KE et al (2016) Differential regulation of translation and endocytosis of alternatively spliced forms of the type II bone morphogenetic protein (BMP) receptor. Mol Biol Cell 27, 716-730

41. Lane KB, Machado RD, Pauciulo MW et al (2000) Heterozygous germline mutations in BMPR2, encoding a TGF-beta receptor, cause familial primary pulmonary 
hypertension. Nat Genet 26, 81-84

42. Deng Z, Morse JH, Slager SL et al (2000) Familial primary pulmonary hypertension (gene PPH1) is caused by mutations in the bone morphogenetic protein receptor-II gene. Am J Hum Genet 67, 737-744

43. Rosenzweig BL, Imamura T, Okadome T et al (1995) Cloning and characterization of a human type II receptor for bone morphogenetic proteins. Proc Natl Acad Sci U S A 92, 7632-7636

44. Sopory S, Nelsen SM, Degnin C, Wong C and Christian JL (2006) Regulation of bone morphogenetic protein-4 activity by sequence elements within the prodomain. J Biol Chem 281, 34021-34031

45. Little SC and Mullins MC (2009) Bone morphogenetic protein heterodimers assemble heteromeric type I receptor complexes to pattern the dorsoventral axis. Nat Cell Biol 11, 637-643

46. Sun $P$, Wang J, Zheng $Y$, Fan $Y$ and Gu Z (2012) BMP2/7 heterodimer is a stronger inducer of bone regeneration in peri-implant bone defects model than BMP2 or BMP7 homodimer. Dent Mater J 31, 239-248

47. Knaus P and Sebald W (2001) Cooperativity of binding epitopes and receptor chains in the BMP/TGFbeta superfamily. Biol Chem 382, 1189-1195

48. Greenwald J, Groppe J, Gray P et al (2003) The BMP7/ ActRII extracellular domain complex provides new insights into the cooperative nature of receptor assembly. Mol Cell 11, 605-617

49. Gilboa L, Nohe A, Geissendorfer T, Sebald W, Henis YI and Knaus P (2000) Bone morphogenetic protein receptor complexes on the surface of live cells: a new oligomerization mode for serine/threonine kinase receptors. Mol Biol Cell 11, 1023-1035

50. Nohe A, Hassel S, Ehrlich M et al (2002) The mode of bone morphogenetic protein (BMP) receptor oligomerization determines different BMP-2 signaling pathways. J Biol Chem 277, 5330-5338

51. Wrana JL, Attisano L, Carcamo J et al (1992) TGF beta signals through a heteromeric protein kinase receptor complex. Cell 71, 1003-1014

52. Koenig BB, Cook JS, Wolsing DH et al (1994) Characterization and cloning of a receptor for BMP-2 and BMP-4 from NIH 3T3 cells. Mol Cell Biol 14, 5961-5974

53. Miyazono K, Maeda S and Imamura T (2005) BMP receptor signaling: transcriptional targets, regulation of signals, and signaling cross-talk. Cytokine Growth Factor Rev 16, 251-263

54. Hassel S, Eichner A, Yakymovych M, Hellman U, Knaus P and Souchelnytskyi S (2004) Proteins associated with type II bone morphogenetic protein receptor (BMPR-II) and identified by two-dimensional gel electrophoresis and mass spectrometry. Proteomics 4, 1346-1358

55. Heldin CH and Moustakas A (2012) Role of Smads in TGFbeta signaling. Cell Tissue Res 347, 21-36

56. Murakami G, Watabe T, Takaoka K, Miyazono K and Imamura T (2003) Cooperative inhibition of bone morphogenetic protein signaling by Smurf1 and inhibitory Smads. Mol Biol Cell 14, 2809-2817

57. Zhu H, Kavsak P, Abdollah S, Wrana JL and Thomsen GH (1999) A SMAD ubiquitin ligase targets the BMP pathway and affects embryonic pattern formation. Nature 400, 687-693

58. Ogata T, Wozney JM, Benezra R and Noda M (1993) Bone morphogenetic protein 2 transiently enhances expression of a gene, Id (inhibitor of differentiation), encoding a helix-loop-helix molecule in osteoblast-like cells. Proc Natl Acad Sci U S A 90, 9219-9222

59. Ogata T and Noda M (1991) Expression of Id, a negative regulator of helix-loop-helix DNA binding proteins, is down-regulated at confluence and enhanced by dexamethasone in a mouse osteoblastic cell line, MC3T3E1. Biochem Biophys Res Commun 180, 1194-1199

60. Katagiri T, Imada M, Yanai T, Suda T, Takahashi N and Kamijo R (2002) Identification of a BMP-responsive element in Id1, the gene for inhibition of myogenesis. Genes Cells 7, 949-960

61. Ito $Y$ and Miyazono K (2003) RUNX transcription factors as key targets of TGF-beta superfamily signaling. Curr Opin Genet Dev 13, 43-47

62. Maeda S, Hayashi M, Komiya S, Imamura T and Miyazono K (2004) Endogenous TGF-beta signaling suppresses maturation of osteoblastic mesenchymal cells. EMBO J 23, 552-563

63. Lee S, Cho HY, Bui HT and Kang D (2014) The osteogenic or adipogenic lineage commitment of human mesenchymal stem cells is determined by protein kinase C delta. BMC Cell Biol 15, 42

64. Satija NK, Gurudutta GU, Sharma S et al (2007) Mesenchymal stem cells: molecular targets for tissue engineering. Stem Cells Dev 16, 7-23

65. Beppu H, Kawabata M, Hamamoto T et al (2000) BMP type II receptor is required for gastrulation and early development of mouse embryos. Dev Biol 221, 249-258

66. Danesh SM, Villasenor A, Chong D, Soukup C and Cleaver O (2009) BMP and BMP receptor expression during murine organogenesis. Gene Expr Patterns 9, 255-265

67. Dyer LA, Pi X and Patterson C (2014) The role of BMPs in endothelial cell function and dysfunction. Trends Endocrinol Metab 25, 472-480

68. Atkinson C, Stewart S, Upton PD et al (2002) Primary pulmonary hypertension is associated with reduced pulmonary vascular expression of type II bone morphogenetic protein receptor. Circulation 105, 1672-1678

69. Finkenzeller G, Hager S and Stark GB (2012) Effects of bone morphogenetic protein 2 on human umbilical vein endothelial cells. Microvasc Res 84, 81-85

70. Langenfeld EM and Langenfeld J (2004) Bone morphogenetic protein-2 stimulates angiogenesis in developing tumors. Mol Cancer Res 2, 141-149

71. Wiley DM, Kim JD, Hao J, Hong CC, Bautch VL and Jin SW (2011) Distinct signalling pathways regulate sprouting angiogenesis from the dorsal aorta and the axial vein. Nat Cell Biol 13, 686-692

72. de Jesus Perez VA, Alastalo TP, Wu JC et al (2009) Bone morphogenetic protein 2 induces pulmonary angiogenesis via Wnt-beta-catenin and Wnt-RhoA-Rac1 pathways. J Cell Biol 184, 83-99

73. Lee KM, Tsai KY, Wang N and Ingber DE (1998) Extracellular matrix and pulmonary hypertension: control of 
vascular smooth muscle cell contractility. Am J Physiol 274, $\mathrm{H} 76-82$

74. Takahashi H, Goto N, Kojima Y et al (2006) Downregulation of type II bone morphogenetic protein receptor in hypoxic pulmonary hypertension. Am J Physiol Lung Cell Mol Physiol 290, L450-458

75. Takahashi K, Kogaki S, Matsushita T, Nasuno S, Kurotobi $\mathrm{S}$ and Ozono K (2007) Hypoxia induces alteration of bone morphogenetic protein receptor signaling in pulmonary artery endothelial cell. Pediatr Res 61, 392-397

76. West J, Harral J, Lane K et al (2008) Mice expressing BMPR2R899X transgene in smooth muscle develop pulmonary vascular lesions. Am J Physiol Lung Cell Mol Physiol 295, L744-755

77. Onishi T, Ishidou Y, Nagamine T et al (1998) Distinct and overlapping patterns of localization of bone morphogenetic protein (BMP) family members and a BMP type II receptor during fracture healing in rats. Bone 22, 605-612

78. Lehnerdt G, Metz KA, Trellakis S, Jahnke K and Neumann A (2007) Signaling by way of type IB and II bone morphogenetic protein receptors regulates bone formation in otospongiosis. Laryngoscope 117, 812-816

79. Garimella R, Kacena MA, Tague SE, Wang J, Horowitz MC and Anderson HC (2007) Expression of bone morphogenetic proteins and their receptors in the bone marrow megakaryocytes of GATA-1(low) mice: a possible role in osteosclerosis. J Histochem Cytochem 55, 745-752

80. Yang C, Yang L, Wan M and Cao X (2010) Generation of a mouse model with expression of bone morphogenetic protein type II receptor lacking the cytoplasmic domain in osteoblasts. Ann N Y Acad Sci 1192, 286-291

81. Katagiri T, Yamaguchi A, Komaki M et al (1994) Bone morphogenetic protein-2 converts the differentiation pathway of $\mathrm{C} 2 \mathrm{C} 12$ myoblasts into the osteoblast lineage. J Cell Biol 127, 1755-1766

82. Hee CK and Nicoll S (2006) Induction of osteoblast differentiation markers in human dermal fibroblasts: potential application to bone tissue engineering. Conf Proc IEEE Eng Med Biol Soc 1, 521-524

83. Davis RL, Weintraub $H$ and Lassar AB (1987) Expression of a single transfected cDNA converts fibroblasts to myoblasts. Cell 51, 987-1000

84. Wright WE, Sassoon DA and Lin VK (1989) Myogenin, a factor regulating myogenesis, has a domain homologous to MyoD. Cell 56, 607-617

85. Edmondson DG and Olson EN (1990) A gene with homology to the myc similarity region of MyoD1 is expressed during myogenesis and is sufficient to activate the muscle differentiation program. Genes Dev 4, 1450

86. Olson EN (1990) MyoD family: a paradigm for develop- ment? Genes Dev 4, 1454-1461

87. Katagiri T, Yamaguchi A, Ikeda T et al (1990) The nonosteogenic mouse pluripotent cell line, $\mathrm{C} 3 \mathrm{H} 10 \mathrm{~T} 1 / 2$, is induced to differentiate into osteoblastic cells by recombinant human bone morphogenetic protein-2. Biochem Biophys Res Commun 172, 295-299

88. Wu N, Zhao Y, Yin Y, Zhang Y and Luo J (2010) Identification and analysis of type II TGF-beta receptors in BMP-9-induced osteogenic differentiation of $\mathrm{C} 3 \mathrm{H} 10 \mathrm{~T} 1 / 2$ mesenchymal stem cells. Acta Biochim Biophys Sin (Shanghai) 42, 699-708

89. Morrell NW (2010) Role of bone morphogenetic protein receptors in the development of pulmonary arterial hypertension. Adv Exp Med Biol 661, 251-264

90. Yang J, Davies RJ, Southwood M et al (2008) Mutations in bone morphogenetic protein type II receptor cause dysregulation of Id gene expression in pulmonary artery smooth muscle cells: implications for familial pulmonary arterial hypertension. Circ Res 102, 1212-1221

91. Hamid R, Hedges LK, Austin E, Phillips JA 3rd, Loyd JE and Cogan JD (2010) Transcripts from a novel BMPR2 termination mutation escape nonsense mediated decay by downstream translation re-initiation: implications for treating pulmonary hypertension. Clin Genet 77, 280-286

92. Ihida-Stansbury K, McKean DM, Lane KB et al (2006) Tenascin- $C$ is induced by mutated BMP type II receptors in familial forms of pulmonary arterial hypertension. Am J Physiol Lung Cell Mol Physiol 291, L694-702

93. Drake KM, Dunmore BJ, McNelly LN, Morrell NW and Aldred MA (2013) Correction of nonsense BMPR2 and SMAD9 mutations by ataluren in pulmonary arterial hypertension. Am J Respir Cell Mol Biol 49, 403-409

94. Nasim MT, Ghouri A, Patel B et al (2008) Stoichiometric imbalance in the receptor complex contributes to dysfunctional BMPR-II mediated signalling in pulmonary arterial hypertension. Hum Mol Genet 17, 1683-1694

95. Howe JR, Bair JL, Sayed MG et al (2001) Germline mutations of the gene encoding bone morphogenetic protein receptor $1 \mathrm{~A}$ in juvenile polyposis. Nat Genet 28, 184-187

96. Kim IY, Lee DH, Lee DK et al (2004) Loss of expression of bone morphogenetic protein receptor type II in human prostate cancer cells. Oncogene 23, 7651-7659

97. Kodach LL, Wiercinska E, de Miranda NF et al (2008) The bone morphogenetic protein pathway is inactivated in the majority of sporadic colorectal cancers. Gastroenterology $134,1332-1341$

98. Schulz TJ and Tseng YH (2009) Emerging role of bone morphogenetic proteins in adipogenesis and energy metabolism. Cytokine Growth Factor Rev 20, 523-531 